Bulletin of the Institute of Mathematics

Academia Sinica (New Series)

Vol. 13 (2018), No. 2, pp. 199-225

DOI: 10.21915 /BIMAS.2018202

\title{
QUANTIZATIONS OF REGULAR FUNCTIONS ON NILPOTENT ORBITS
}

\begin{abstract}
IVAN LOSEV
Dedicated to George Lusztig, on his 70th birthday, with admiration.

Department of Mathematics, Northeastern University, Boston MA 02115 USA.

E-mail: i.loseu@neu.edu

\|\|$\|$

Abstract

We study the quantizations of the algebras of regular functions on nilpotent orbits. We show that such a quantization always exists and is unique if the orbit is birationally rigid. Further we show that, for special birationally rigid orbits, the quantization has integral central character in all cases but four (one orbit in $E_{7}$ and three orbits in $E_{8}$ ). We use this to complete the computation of Goldie ranks for primitive ideals with integral central character for all special nilpotent orbits but one (in $E_{8}$ ). Our main ingredient are results on the geometry of normalizations of the closures of nilpotent orbits by $\mathrm{Fu}$ and Namikawa.
\end{abstract}

\section{Introduction}

\subsection{Nilpotent orbits and their quantizations}

Let $G$ be a connected semisimple algebraic group over $\mathbb{C}$ and let $\mathfrak{g}$ be its Lie algebra. Pick a nilpotent orbit $\mathbb{O} \subset \mathfrak{g}$. This orbit is a symplectic algebraic variety with respect to the Kirillov-Kostant form. So the algebra $\mathbb{C}[\mathbb{O}]$ of regular functions on $\mathbb{O}$ acquires a Poisson bracket. This algebra is also naturally graded and the Poisson bracket has degree -1 . So one can ask about quantizations of $\mathbb{O}$, i.e., filtered algebras $\mathcal{A}$ equipped with an isomorphism gr $\mathcal{A} \stackrel{\sim}{\longrightarrow} \mathbb{C}[\mathbb{O}]$ of graded Poisson algebras.

Received May 15, 2016 and in revised form June 30, 2016.

AMS Subject Classification: Primary 17B35; Secondary 53D55, 16 G99.

Key words and phrases: Nilpotent orbit, quantization, primitive ideal, W-algebra.

Supported by the NSF grant DMS-1161584. 
We are actually interested in quantizations that have some additional structures mirroring those of $\mathbb{O}$. Namely, the group $G$ acts on $\mathbb{O}$ and the inclusion $\mathbb{O} \hookrightarrow \mathfrak{g}$ is a moment map for this action. We want the $G$-action on $\mathbb{C}[\mathbb{O}]$ to lift to a filtration preserving action on $\mathcal{A}$. Further, we want a $G$-equivariant homomorphism $U(\mathfrak{g}) \rightarrow \mathcal{A}$ such that, for any $\xi \in \mathfrak{g}$, the endomorphism $[\xi, \cdot]: \mathcal{A} \rightarrow \mathcal{A}$ coincides with the differential of the $G$-action (in other words, $\mathcal{A}$ has to be a Dixmier algebra in the sense of Vogan, [40]). A motivation to consider quantizations of $\mathbb{C}[\mathbb{O}]$ of this form comes from attempts to extend the orbit method to reductive groups, see, e.g., 30] for details, where quantizations of $\mathbb{C}[\mathbb{O}]$ for certain orbits $\mathbb{O}$ in classical Lie algebras are constructed.

We establish the existence of such a quantization $\mathcal{A}$ and we investigate the questions of when $\mathcal{A}$ is unique and when the kernel of the map $U(\mathfrak{g}) \rightarrow$ $\mathcal{A}$ has integral central character. The latter question is of importance for computing Goldie ranks of primitive ideals with integral central characters in exceptional algebras (the case of classical Lie algebras was settled in [23]). We elaborate on our results in the next section.

The questions above are closely related to the representation theory of finite W-algebras introduced by Premet in [36], see [19, 33, 41] for reviews. Each $\mathrm{W}$-algebra is constructed from a pair $(\mathfrak{g}, \mathbb{O})$ of a semisimple Lie algebra $\mathfrak{g}$ and a nilpotent orbit $\mathbb{O} \subset \mathfrak{g}$. Dixmier algebras quantizing $\mathbb{C}[\mathbb{O}]$ are closely related to one-dimensional modules (with certain additional properties) over the W-algebra constructed from $(\mathfrak{g}, \mathbb{O})$.

\subsection{Main results}

Here are three lists of nilpotent orbits in exceptional Lie algebras that are all exceptional in some further ways explained below in the paper.

(e1) $\tilde{A}_{1}$ in $G_{2}, \tilde{A}_{2}+A_{1}$ in $F_{4},\left(A_{3}+A_{1}\right)^{\prime}$ in $E_{7}, A_{3}+A_{1}, A_{5}+A_{1}, D_{5}\left(a_{1}\right)+A_{2}$ in $E_{8}$.

(e2) $A_{4}+A_{1}$ in $E_{7}, A_{4}+A_{1}, E_{6}\left(a_{1}\right)+A_{1}$ in $E_{8}$.

(e3) $A_{4}+2 A_{1}$ in $E_{8}$. 
We note that the three orbits in (e2) are exceptional in the sense of Lusztig, [26].

Theorem 1.1. The following is true.

(1) For any nilpotent orbit $\mathbb{O}$, there is a Dixmier algebra $\mathcal{A}$ quantizing $\mathbb{C}[\mathbb{O}]$.

(2) If $\mathbb{O}$ is birationally rigid (see Section 2.3 below for a definition), then $\mathcal{A}$ in (1) is unique.

(3) If (O) is special but not one of the four orbits in (e2),(e3), then $\mathcal{A}$ from (ii) has integral central character. For orbits (e2) and (e3), $\mathcal{A}$ does not have integral central character.

In the subsequent paper 24] we will classify all filtered quantizations of $\mathbb{C}[\mathbb{O}]$.

Corollary 1.2. Let $\mathcal{J}$ be a primitive ideal in $U(\mathfrak{g})$ with integral central character. Suppose that the associated orbit is not as in (e3). Then the Goldie rank of $\mathcal{J}$ coincides with the dimension of the corresponding $W$-algebra module.

In [23] we have obtained basically Kazhdan-Lusztig type formulas for the dimensions of the irreducible finite dimensional $\mathrm{W}$-algebra modules with integral central character. So we can view Corollary 1.2 as a formula for the Goldie ranks. This corollary has been already proved for classical types in [23, Theorem 1.2].

\subsection{Content of the paper}

We start by recalling various properties of nilpotent orbits: their classification, the notion of a special orbit, Lusztig-Spaltenstein induction and (birationally) rigid orbits, the structure of the boundary, and $\mathbb{Q}$-factorial terminalizations.

In Section 3 we recall some known results about $\mathrm{W}$-algebras. First, we recall their definition following the approach taken in [16] and refined in further papers by the author, see, e.g., 22]. Then we recall an important construction from [17]: functors between the categories of Harish-Chandra bimodules. After that we recall the category $\mathcal{O}$ from [5] and related constructions. Next, we recall the classification, 25], of finite dimensional irreducible 
modules with integral central characters over W-algebras. Finally, we explain some constructions and results related to quantizations of symplectic varieties, including nilpotent orbits.

In Section 4 we prove our main results. An important auxiliary result is Theorem 4.4 that gives a sufficient condition for a functor $\bullet \nmid$ from [17] between suitable categories of bimodules to be an equivalence. We also derive some corollaries, see Section 4.3.

\section{Preliminaries on Nilpotent Orbits}

\subsection{Classification and special orbits}

First, let us recall the classification of nilpotent orbits in semisimple Lie algebras. The nilpotent orbits in $\mathfrak{s l}_{n}$ are classified by the partitions of $n$. We sometimes will write partitions as $m^{d_{m}}(m-1)^{d_{m-1}} \ldots 1^{d_{1}}$, where superscripts indicate multiplicities. The nilpotent orbits in $\mathfrak{s o}_{2 n+1}$ are classified by the partitions of $2 n+1$ that have type $B$ meaning that every even part appears even number of times (to an orbit we assign its Jordan type in the tautological representation of dimension $2 n+1$ ). The nilpotent orbits in $\mathfrak{s p}_{2 n}$ are classified by partitions of $2 n$ that have type $C$ meaning that every odd part appears even number of times. The nilpotent orbits of $\mathrm{O}_{2 n}$ in $\mathfrak{s o}_{2 n}$ are classified by partitions of $2 n$ of type $D$ meaning that every even part appears even number of times. The $\mathrm{SO}_{2 n}$-action on the $\mathrm{O}_{2 n}$-orbit corresponding to a partition $\mu$ is transitive if and only if there is an odd part in $\mu$, otherwise the $\mathrm{O}_{2 n}$-orbit splits into the union of two $\mathrm{SO}_{2 n}$-orbits. For a partition $\mu$, we will write $\mathbb{O}_{\mu}$ for the corresponding orbit (in the case of $\mathfrak{g}=\mathfrak{s o}_{2 n}$ we consider orbits for $\mathrm{O}_{2 n}$ ).

The classification in the exceptional types is also known, there the orbits have labels consisting of a Dynkin diagram type (e.g., $A_{3}+A_{2}$ ), sometimes with an additional decoration. The Dynkin diagram is that of a minimal Levi subalgebra containing an element of a given orbit.

Below we will need some information about so called special orbits (as defined by Lusztig, [26, 13.1.1]). All orbits in type A are special. An orbit in type $B$ or $C$ corresponding to a partition $\mu$ is special if $\mu^{t}$ has type $B$ or $C$, respectively. An orbit in type $D$ is special if $\mu^{t}$ has type $C$. Special orbits 
in the exceptional algebras have been classified as well, see [6, Section 13.4] or [7, Section 8.4].

There is an order reversing bijection $\mathbf{d}$ (called the Barbasch-VoganSpaltenstein duality, a somewhat implicit construction was earlier discovered by Lusztig) between the sets of special orbits in $\mathfrak{g}$ and in the Langlands dual algebra ${ }^{L} \mathfrak{g}$. For the classical types, it is described combinatorially, see [7, Section 6.3], we will not need this description. The description of this duality in exceptional types is provided in [6, Section 13.4].

\subsection{Structure of the boundary}

Below we will need some information about singularities of the closures $\overline{\mathbb{O}}$. We start by studying the situation when $\operatorname{codim}_{\overline{\mathbb{O}}} \partial \mathbb{O} \geqslant 4$.

The following claim is [35, Proposition 1.3.2].

Lemma 2.1. Suppose $\mathfrak{g}$ is classical. For $\mathbb{O}=\mathbb{O}_{\mu}$, the inequality $\operatorname{codim}_{\overline{\mathbb{O}}} \partial \mathbb{O}$ $\geqslant 4$ is equivalent to $\mu_{i}-\mu_{i+1} \leqslant 1$ for all $i$.

The following is the list of the orbits $\mathbb{O}$ in exceptional Lie algebras that satisfy $\operatorname{codim}_{\overline{\mathbb{O}}} \partial \mathbb{O} \geqslant 4$. This information can be extracted, for example, from [11, Section 13].

$G_{2}: A_{1}$.

$F_{4}: A_{1}, \tilde{A}_{1}, A_{1}+\tilde{A}_{1}, A_{2}+\tilde{A}_{1}$.

$E_{6}: A_{1}, 2 A_{1}, 3 A_{1}, A_{2}+A_{1}, A_{2}+2 A_{1}, 2 A_{2}+A_{1}$.

$E_{7}: A_{1}, 2 A_{1},\left(3 A_{1}\right)^{\prime}, 4 A_{1}, A_{2}+A_{1}, A_{2}+2 A_{1}, 2 A_{2}+A_{1}, A_{4}+A_{1}$.

$E_{8}: A_{1}, 2 A_{1}, 3 A_{1}, 4 A_{1}, A_{2}+A_{1}, A_{2}+2 A_{1}, A_{2}+3 A_{1}, 2 A_{2}+A_{1}, 2 A_{2}+2 A_{1}, A_{3}+$ $2 A_{1}, A_{3}+A_{2}+A_{1}, A_{4}+A_{1}, 2 A_{3}, A_{4}+2 A_{1}, D_{4}\left(a_{1}\right)+A_{1}, A_{4}+A_{3}$.

Let us proceed to explaining results about the structure of the boundary of the normalization $\operatorname{Spec}(\mathbb{C}[\mathbb{O}])$.

Lemma 2.2. The codimension of $\operatorname{Spec}(\mathbb{C}[\mathbb{O}])^{\text {reg }} \backslash \mathbb{O}$ in $\operatorname{Spec}(\mathbb{C}[\mathbb{O}])$ is $\geqslant 4$ for all but the following cases:

$G_{2}: \tilde{A}_{1}$,

$F_{4}: \tilde{A}_{2}+A_{1}, C_{3}\left(a_{1}\right)$, 
$E_{6}: A_{3}+A_{1}$,

$E_{7}:\left(A_{3}+A_{1}\right)^{\prime}, D_{6}\left(a_{2}\right)$,

$E_{8}: A_{3}+A_{1}, A_{5}+A_{1}, D_{5}\left(a_{1}\right)+A_{2}, D_{6}\left(a_{2}\right), E_{6}\left(a_{3}\right)+A_{1}, E_{7}\left(a_{2}\right), E_{7}\left(a_{5}\right)$.

Here and below the superscript "reg" means the smooth locus.

Proof. The smooth locus in $\overline{\mathbb{O}}$ coincides with $\mathbb{O}$. So the inequality codim $\operatorname{Spec}(\mathbb{C}[\mathbb{O}])^{r e g} \backslash \mathbb{O} \geqslant 4$ is equivalent to the claim that, for any orbit $\mathbb{O}^{\prime}$ of codimension 2 in $\overline{\mathbb{O}}$, the normalization of a formal slice to $\mathbb{O}^{\prime}$ in $\mathbb{O}$ is not smooth. By results of [14, 15], this is always true when $\mathfrak{g}$ is classical. Now we consider exceptional algebras. By results of [11], the equality $\operatorname{codim} \operatorname{Spec}(\mathbb{C}[\mathbb{O}])^{r e g} \backslash \mathbb{O}=2$ is equivalent to at least one edge going down from the label of $\mathbb{O}$ in the graphs of Section 14 being marked with an "m". Examining these tables we get a required result.

\subsection{Induction and birational induction}

Let $\mathfrak{l}$ be a Levi subalgebra in $\mathfrak{g}$ and $\mathbb{O}^{\prime} \subset \mathfrak{l}$ be a nilpotent orbit. Pick a parabolic subalgebra $\mathfrak{p} \subset \mathfrak{g}$ with Levi subalgebra $\mathfrak{l}$ and let $\mathfrak{n}$ denote the nilpotent radical of $\mathfrak{p}$. Let $P \subset G$ denote the corresponding parabolic subgroup. Then the fiber bundle $G *_{P}\left(\overline{\mathbb{O}}^{\prime} \times \mathfrak{n}\right)$ naturally maps to $\mathcal{N}$, this is known as the generalized Springer map. Obviously, there is a unique dense orbit $\mathbb{O}$ in the image. This orbit is called induced from $\mathbb{O}^{\prime}$ (see [28]), in fact, it is independent of the choice of $\mathfrak{p}$. If the map $G *_{P}\left(\overline{\mathbb{O}}^{\prime} \times \mathfrak{n}\right) \rightarrow \overline{\mathbb{O}}$ is birational, then we say that $\mathbb{O}$ is birationally induced from $\mathbb{O}^{\prime}$. An orbit that cannot be (birationally) induced from an orbit in a proper Levi is called (birationally) rigid.

The induction for classical Lie algebras can be described combinatorially on the level of partitions. We say that a partition $\mu$ of type $X$ (where $X$ is $B, C$ or $D)$ is obtained from a partition $\mu^{\prime}$ by an elementary step if

(i) either there is $n$ such that $\mu_{i}=\mu_{i}^{\prime}+2$ for $i \leqslant n$, and $\mu_{i}=\mu_{i}^{\prime}$ for $i>n$,

(ii) or there is $n$ such that $\mu_{i}=\mu_{i}^{\prime}+2$ for $i<n, \mu_{n}=\mu_{n}^{\prime}+1, \mu_{n+1}=$ $\mu_{n+1}^{\prime}+1, \mu_{i}=\mu_{i}^{\prime}$ for $i>n+1$, and the partition obtained from $\mu^{\prime}, n$ in (i) does not have a correct type. 
The orbit $\mathbb{O}_{\mu} \subset \mathfrak{s o}|\mu|$ is induced from the orbit $\mathbb{O}_{\left|\mu^{\prime}\right|} \times\{0\} \subset \mathfrak{s o}_{\left|\mu^{\prime}\right|} \times \mathfrak{g l}_{?} \times \ldots \mathfrak{g l}$ ? if and only $\mu$ is obtained from $\mu^{\prime}$ by a sequence of elementary steps (and the same is true for $\mathfrak{s p}$ 's).

Proposition 2.3. Any special orbit can be birationally induced from a birationally rigid special orbit.

Proof. Classical types. We claim that the induction is birational if and only if only elementary steps of type (i) are involved. The "if" part is established in [23, 5.4.2-5.4.4]. To establish the "only if" part, we just need to show that, for an elementary step of type (ii), the generalized Springer map is not birational.

Let us consider the orthogonal case, the symplectic one is similar. Note that in (ii), the numbers $\mu_{n}^{\prime}$ and $\mu_{n+1}^{\prime}$ coincide and are even. The orbit $\mathbb{O}_{\mu}$ is induced from $\mathbb{O}_{\mu^{\prime}} \times\{0\} \subset \mathfrak{s a}_{m} \times \mathfrak{g l}_{n}$. Recall that $P$ acts transitively on $\left(\mathbb{O}^{\prime} \times \mathfrak{n}\right) \cap \mathbb{O}$, see [28, Theorem 1.3]. So if we know that $Z_{G}(e) \not \subset Z_{P}(e)$ for a particular choice of $e$ in $\left(\mathbb{O}^{\prime} \times \mathfrak{n}\right) \cap \mathbb{O}$, then the corresponding induction is not birational. Split $\mathbb{C}^{m}$ into the sum $\left(U \oplus U^{*}\right) \oplus V^{\prime}$, where $\operatorname{dim} U=\mu_{n}^{\prime}$, the subspaces $U \oplus U^{*}$ and $V^{\prime}$ are orthogonal to one another, and $U$ is lagrangian in $U \oplus U^{*}$. We can then split $\mathbb{C}^{m+2 n}$ as $\left(\tilde{U} \oplus \tilde{U}^{*}\right) \oplus \tilde{V}$ with $\tilde{U}=U \oplus \mathbb{C}$. We have an element in $\left(\mathbb{O}^{\prime} \times \mathfrak{n}\right) \cap \mathbb{O}$ of the form $e_{1}+e_{2}$, where $e_{1} \in \mathfrak{s o}\left(\tilde{V}^{\prime}\right)$ and $e_{2} \in \mathfrak{g l}(\tilde{U}) \hookrightarrow \mathfrak{s o}\left(\tilde{U} \oplus \tilde{U}^{*}\right)$ is a single Jordan block. Then the summand $\mathbb{C} \subset \tilde{U}$ is the kernel of $e_{2}$. But note that the centralizer of $e_{2} \in \mathfrak{s o}\left(\tilde{U} \oplus \tilde{U}^{*}\right)$ does not preserve the kernel of $e_{2}$ in $\tilde{U}$. This finishes the argument for $\mathfrak{g}$ of orthogonal type.

So all "weakly rigid" orbits listed in [23, 5.4.2-5.4.4] are birationally rigid. Since any special orbit can be birationally induced from one of weakly rigid orbits, our claim follows.

Exceptional types. We proceed by the induction on $\mathrm{rk} \mathfrak{g}$. We need to show that if a special orbit is not birationally rigid, then it can be birationally induced from a special orbit.

First, we consider the case when the group $A(\mathbb{O})$ is trivial. Here any induction is birational, so $\mathbb{O}$ is birationally rigid if and only if it is rigid. By [7, Theorem 8.3.1], if the dual of $\mathbb{O}$ intersects a Levi subalgebra, then (1) is properly induced from a special orbit. So we only need to consider orbits whose duals are distinguished (and are not distinguished themselves, 
those are obviously induced). The duality is described in [6, Section 13.4]. Examining the data from there we arrive at the following list of orbits whose duals are distinguished and that are not distinguished (regardless of $A(\mathbb{O})$ ):

$\left(G_{2}\right)$ None.

(F) $\tilde{A}_{1}, \tilde{A}_{1}+A_{1}$.

(E) $A_{1}, A_{2}$.

(E) $A_{1}, 2 A_{1}, A_{2}, A_{2}+2 A_{1}, D_{4}\left(a_{1}\right)$.

(E) $A_{1}, 2 A_{1}, A_{2}, A_{2}+A_{1}, A_{2}+2 A_{1}, 2 A_{2}, D_{4}\left(a_{1}\right), D_{4}\left(a_{1}\right)+A_{1}, D_{4}\left(a_{1}\right)+A_{2}$.

According to tables in [7, Section 8.4], the orbits $\tilde{A}_{1}$ in $F_{4}, A_{2}$ in $E_{6}$, $A_{2}, D_{4}\left(a_{1}\right)$ in $E_{7}, A_{2}, A_{2}+A_{1}, 2 A_{2}, D_{4}\left(a_{1}\right), D_{4}\left(a_{1}\right)+A_{1}, D_{4}\left(a_{1}\right)+A_{2}$ in $E_{8}$ have nontrivial groups $A(\mathbb{O})$. The remaining orbits in the list above are rigid, see, e.g., [8, Section 4].

Let us proceed to the case of nontrivial $A(\mathbb{O})$. According to $[10$, Section 3] combined with the lists of special orbits, see, e.g., [6, Section 13.4] or [7, Section 8.4], the following special orbits have nontrivial $A(\mathbb{O})$ and are not birationally induced from a zero orbit.

$\left(G_{2}\right)$ None.

$\left(F_{4}\right)$ None.

$\left(E_{6}\right)$ None.

(E $\left.E_{7}\right) A_{2}+A_{1}, A_{3}+A_{2}, A_{4}+A_{1}, D_{5}\left(a_{1}\right)$.

(E) $A_{3}+A_{2}, A_{4}+A_{1}, A_{4}+2 A_{1}, D_{5}\left(a_{1}\right), E_{6}\left(a_{1}\right)+A_{1}, E_{7}\left(a_{3}\right), E_{7}\left(a_{4}\right)$.

The orbits $A_{2}+A_{1}, A_{4}+A_{1}$ in $E_{7}$, and $A_{4}+A_{1}, A_{4}+2 A_{1}$ in $E_{8}$ are birationally rigid by [10, Proposition 3.1].The orbit $A_{3}+A_{2}$ in $E_{7}$ is birationally induced from the orbit $\left(2^{2} 1^{6}, 1^{2}\right)$ in $D_{5}+A_{1}$ that is special. The orbit $D_{5}\left(a_{1}\right)$ is birationally induced from the orbit $3^{2} 2^{2} 1^{2}$ in $D_{6}$ which is also special. These computations were done in [10, Section 3.3].

Let us proceed to the remaining 5 orbits in $E_{8}$ following [10, Section 3.4]. The orbit $A_{3}+A_{2}$ is birationally induced from the special orbit $2^{2} 1^{10}$ in $D_{7}$. The orbit $D_{5}\left(a_{1}\right)$ is birationally induced from the special orbit $A_{2}+A_{1}$ in $E_{7}$. The orbit $E_{6}\left(a_{1}\right)+A_{1}$ is birationally induced from the special orbit $A_{4}+A_{1}$ in $E_{7}$. The orbit $E_{7}\left(a_{3}\right)$ is birationally induced from the special 
orbit $3^{2} 2^{2} 1^{2}$ in $D_{6}$. Finally, the orbit $E_{7}\left(a_{4}\right)$ is birationally induced from the special orbit $A_{3}+A_{2}$ in $E_{7}$.

Note that the proof implies that an orbit $\mathbb{O}_{\mu}$ in a classical Lie algebra is birationally rigid if and only if $\mu$ satisfies the combinatorial condition of Lemma 2.1. Note also that the orbits $A_{2}+A_{1}, A_{4}+A_{1}$ in $E_{7}$ and $A_{4}+$ $A_{1}, A_{4}+2 A_{1}$ in $E_{8}$ are only birationally rigid but not rigid orbits in the exceptional Lie algebras, see [10, Proposition 3.1]. This fact together with the classification of rigid orbits, see, e.g., [8, Section 4], and Lemma2.2 imply the following claim.

Lemma 2.4. The only birationally rigid orbits that fail the condition of Lemma 2.2 are the six orbits from (e1).

\section{4. $\mathbb{Q}$-factorial terminalizations}

Here we are going to recall a result of [35] and [10] and explain some corollaries. This result (proved in [35] for the classical types, in [10] for the exceptional types, and in [24, Proposition 4.4] conceptually) can be stated as follows.

Proposition 2.5. Let $\mathbb{O}$ be a birationally rigid orbit. Then $\operatorname{Spec}(\mathbb{C}[\mathbb{O}])$ has $\mathbb{Q}$-factorial terminal singularities.

Now let $\mathbb{O}$ be an arbitrary orbit. Suppose that $\mathbb{O}$ is birationally induced from $\mathbb{O}^{\prime} \subset \mathfrak{l}$. Then the morphism $G *_{P}\left(\operatorname{Spec}\left(\mathbb{C}\left[\mathbb{O}^{\prime}\right]\right) \times \mathfrak{n}\right) \rightarrow \operatorname{Spec}(\mathbb{C}[\mathbb{O}])$ (obtained by lifting the generalized Springer map to the normalizations) is a $\mathbb{Q}$-factorial terminalization.

Also recall the following classical result, see, e.g., [9, Corollary 2.10].

Proposition 2.6. For any $\mathbb{O}$, the variety $\operatorname{Spec}(\mathbb{C}[\mathbb{O}])$ has symplectic singularities in the sense of Beauville.

Let us deduce some corollaries from these results. The following result can be proved along the lines of the proof of [34, Lemma 12].

Corollary 2.7. Let $\mathbb{O}^{\prime} \subset \mathfrak{l}$ be a birationally rigid orbit and let $\mathbb{O} \subset \mathfrak{g}$ be birationally induced from $\mathbb{O}^{\prime}$. Let $X:=G *_{P}\left(\operatorname{Spec}\left(\mathbb{C}\left[\mathbb{O}^{\prime}\right]\right) \times \mathfrak{n}\right)$. Then $H^{i}\left(X^{r e g}, \mathcal{O}_{X^{r e g}}\right)=0$ for $i=1,2$. 
The next claim follows from Corollary 2.7 and Lemma 2.4.

Corollary 2.8. Let $\mathbb{O}$ be a birationally rigid orbit that is not one of the six orbits listed in Lemma 2.4. Then $H^{i}\left(\mathbb{O}, \mathcal{O}_{\mathbb{O}}\right)=0$ for $i=1,2$.

We will need one more result about nilpotent orbits.

Lemma 2.9. For any nilpotent orbit $\mathbb{O}$, we have $H_{D R}^{1}(\mathbb{O})=\{0\}$. If $\mathbb{O}$ is birationally rigid, then $H_{D R}^{2}(\mathbb{O})=\{0\}$.

Proof. To prove that $H_{D R}^{1}(\mathbb{O})=\{0\}$, we note that the fundamental group of $\mathbb{( 1 )}$ is finite.

Now let us consider the case of birationally rigid orbits. Since $\operatorname{Spec}(\mathbb{C}[\mathbb{O}])$ is $\mathbb{Q}$-factorial, we conclude that the group $\operatorname{Hom}\left(Z_{G}(e), \mathbb{C}^{\times}\right)$is finite. So $\left(\mathfrak{q}^{*}\right)^{Q}$ is zero. On the other hand, a standard argument shows that this space is $H_{D R}^{2}(\mathbb{O})$.

\section{Preliminaries on $\mathrm{W}$-algebras and Quantizations}

\subsection{W-algebras}

Let $G$ be a reductive algebraic group, $\mathfrak{g}$ its Lie algebra. Pick a nilpotent orbit $\mathbb{O} \subset \mathfrak{g}$. Choose an element $e \in \mathbb{O}$ and include it into an $\mathfrak{s l}_{2}$-triple $(e, h, f)$. We write $Q$ for the centralizer of $(e, h, f)$ in $G$.

From the triple $(e, h, f)$ we can produce a filtered associative algebra $\mathcal{W}$ equipped with a Hamiltonian $Q$-action. Namely, consider the universal enveloping algebra $\mathcal{U}=U(\mathfrak{g})$ with its standard PBW filtration $\mathcal{U}=\bigcup_{i \geqslant 0} \mathcal{U}_{\leqslant i}$. It will be convenient for us to double the filtration and set $F_{i} \mathcal{U}:=\mathcal{U}_{\leqslant[i / 2]}$. Form the Rees algebra $\mathcal{U}_{\hbar}:=\bigoplus_{i}\left(F_{i} \mathcal{U}\right) \hbar^{i}$. The quotient $\mathcal{U}_{\hbar} /(\hbar)$ coincides with $S(\mathfrak{g})=\mathbb{C}\left[\mathfrak{g}^{*}\right]$. Identify $\mathfrak{g}$ with $\mathfrak{g}^{*}$ by means of the Killing form and let $\chi \in \mathfrak{g}^{*}$ be the image of $e$. Consider the completion $\mathcal{U}_{\hbar}^{\wedge}$ in the topology induced by the preimage of the maximal ideal of $\chi$. The space $V:=[\mathfrak{g}, f]$ is symplectic with the form given by $\langle\chi,[\cdot, \cdot]\rangle$. So we can form the homogenized Weyl algebra $\mathbb{A}_{\hbar}$ of $V$, that is, the Rees algebra of the usual Weyl algebra $\mathbb{A}(V)$. We consider the completion $\mathbb{A}_{\hbar}^{\wedge_{0}}$ in the topology induced by the maximal ideal of $0 \in V$. Both $\mathcal{U}_{\hbar}^{\wedge}$ and $\mathbb{A}_{\hbar}^{\wedge}$ come equipped with actions of $Q \times \mathbb{C}^{\times}$. The action of $Q$ on $\mathcal{U}_{\hbar}^{\wedge}, \mathbb{A}_{\hbar}^{\wedge_{0}}$ is induced from the natural actions of $Q$ on $\mathfrak{g}$ and $V$, respectively. The group $\mathbb{C}^{\times}$acts on $\mathfrak{g}^{*}$ via $t . \alpha:=t^{-2} \gamma(t) \alpha$, where 
$\gamma: \mathbb{C}^{\times} \rightarrow G$ is the one-parameter subgroup associated to $h$. It acts on $V$ by $t . v:=\gamma(t)^{-1} v$. Finally, we set $t . \hbar:=t \hbar$, this defines $\mathbb{C}^{\times}$-actions on $\mathcal{U}_{\hbar}^{\wedge} \chi, \mathbb{A}_{\hbar}^{\wedge_{0}}$ by topological algebra automorphisms that commute with the $Q$-actions.

It was checked in [16, Section 3.3], see also [22, Section 2.2], that there is a $Q \times \mathbb{C}^{\times}$-equivariant $\mathbb{C}[\hbar]$-linear embedding $\mathbb{A}_{\hbar}^{\wedge_{0}} \hookrightarrow \mathcal{U}_{\hbar}^{\wedge}$ such that we have the decomposition

$$
\mathcal{U}_{\hbar}^{\wedge \chi} \cong \mathbb{A}_{\hbar}^{\wedge_{0}} \widehat{\otimes}_{\mathbb{C}[[\hbar]]} \mathcal{W}_{\hbar}^{\prime}
$$

where we write $\mathcal{W}_{\hbar}^{\prime}$ for the centralizer of $\mathbb{A}_{\hbar}^{\wedge_{0}}$ in $\mathcal{U}_{\hbar}^{\wedge}$. This centralizer comes with an action of $Q \times \mathbb{C}^{\times}$. Let us write $\mathcal{W}_{\hbar}$ for the $\mathbb{C}^{\times}$-finite part of $\mathcal{W}_{\hbar}^{\prime}$, then $\mathcal{W}_{\hbar}^{\prime}$ is naturally identified with the completion $\mathcal{W}_{\hbar}^{\wedge}$. Set $\mathcal{W}:=\mathcal{W}_{\hbar} /(\hbar-1)$. This is a filtered algebra with a Hamiltonian $Q$-action that does not depend on the choice of the embedding $\mathbb{A}_{\hbar}^{\wedge_{0}} \hookrightarrow \mathcal{U}_{\hbar}^{\wedge} \chi$ up to an isomorphism preserving the filtration and the action. See [22, Section 2.1]. The associated graded algebra gr $\mathcal{W}$ coincides with $\mathbb{C}[S]$, where $S:=e+\operatorname{ker}$ ad $f$ is the Slodowy slice.

By a result of Ginzburg, which is a footnote in [37, Section 5.7], the natural map $Z(\mathcal{U}) \rightarrow Z(\mathcal{W})$ (where $Z$ stands for the center) is an isomorphism. So we can speak, for example, about $\mathcal{W}$-modules with integral central character.

\subsection{Functors between Harish-Chandra bimodules}

By a $G$-equivariant Harish-Chandra $\mathcal{U}$-bimodule (or $(\mathcal{U}, G)$-module) we mean a finitely generated $\mathcal{U}$-bimodule $\mathcal{B}$ such that the adjoint $\mathfrak{g}$-action is locally finite and integrates to an action of $G$. We can also introduce the notion of a $Q$-equivariant $\mathrm{HC} \mathcal{W}$-bimodule, see [17, Section 2.5]. We write $\mathrm{HC}^{G}(\mathcal{U}), \mathrm{HC}^{Q}(\mathcal{W})$ for the categories of equivariant $\mathrm{HC}$ bimodules.

In [17], we have constructed an exact functor $\bullet$ : $\mathrm{HC}^{G}(\mathcal{U}) \rightarrow \mathrm{HC}^{Q}(\mathcal{W})$. Let us recall the construction of the functor. Pick a $G$-equivariant $\mathrm{HC}$ bimodule $\mathcal{B}$ and equip it with a good filtration compatible with the filtration $\mathrm{F}_{i} \mathcal{U}$. So the Rees $\mathbb{C}[\hbar]$-module $\mathcal{B}_{\hbar}:=R_{\hbar}(\mathcal{B})$ is a $G$-equivariant $\mathcal{U}_{\hbar}$-bimodule. Consider the completion $\mathcal{B}_{\hbar}^{\wedge}$ in the $\chi$-adic topology. This is a $Q \times \mathbb{C}^{\times}$-equivariant $\mathcal{U}_{\hbar}^{\wedge}$-bimodule (the action of $Q$ is Hamiltonian, while the action of $\mathbb{C}^{\times}$is not). As was checked in [17, Proposition 3.3.1], $\mathcal{B}_{\hbar}^{\wedge \chi}=\mathbb{A}_{\hbar}^{\wedge_{0}} \widehat{\otimes}_{\mathbb{C}[[\hbar]]} \underline{\mathcal{B}}_{\hbar}^{\prime}$, where $\underline{\mathcal{B}}_{\hbar}^{\prime}$ is the centralizer of $\mathbb{A}_{\hbar}^{\wedge_{0}}$. So $\underline{\mathcal{B}}_{\hbar}^{\prime}$ is a $Q \times \mathbb{C}^{\times}$-equivariant $\mathcal{W}_{\hbar}^{\wedge}$-bimodule. One 
can show, see loc. cit., that it coincides with the completion of its $\mathbb{C}^{\times}$-finite part $\underline{\mathcal{B}}_{\hbar}$. We set $\mathcal{B}_{\dagger}:=\underline{\mathcal{B}}_{\hbar} /(\hbar-1)$. This is an object in $\mathrm{HC}^{Q}(\mathcal{W})$ that comes equipped with a good filtration. This filtration depends on the choice of a filtration on $\mathcal{B}$, while $\mathcal{B}_{\dagger}$ itself does not.

Let us list properties of the functor $\bullet \nmid$ established in [17, Sec. 3.3,3.4].

Lemma 3.1. The following is true:

(1) $\mathcal{U}_{\dagger}=\mathcal{W}$

(2) $\bullet$ is an exact functor.

(3) $\bullet$ intertwines the tensor products.

(4) $\operatorname{gr} \mathcal{B}_{\dagger}$ (with respect to the filtration above) coincides with the pull-back of gr $\mathcal{B}$ to $S$.

(5) In particular, $\bullet$ maps the category $\mathrm{HC}_{\overline{\mathbb{O}}}(\mathcal{U})$ of all $H C$ bimodules supported on $\overline{\mathbb{O}}$ to the category $\mathrm{HC}_{\text {fin }}^{Q}(\mathcal{W})$ of all finite dimensional $Q$-equivariant $\mathcal{W}$-bimodules. Further, $\bullet \dagger$ annihilates $\mathrm{HC}_{\partial \mathbb{O}}^{G}(\mathcal{U})$.

(6) The induced functor $\mathrm{HC}_{\mathbb{O}}^{G}(\mathcal{U}):=\mathrm{HC}_{\overline{\mathbb{O}}}^{G}(\mathcal{U}) / \mathrm{HC}_{\partial \mathbb{O}}^{G}(\mathcal{U}) \rightarrow \mathrm{HC}_{\text {fin }}^{Q}(\mathcal{W})$ is a full embedding whose image is closed under taking subquotients.

(7) The functor $\bullet$ respects central characters on the left and on the right.

(8) For $\mathcal{B} \in \mathrm{HC}_{\overline{\mathbb{O}}}(\mathcal{U})$, the dimension of $\mathcal{B}_{\dagger}$ coincides with the multiplicity of $\mathcal{B}$ on $\mathbb{O}$.

The functor $\bullet \nmid: \mathrm{HC}_{\overline{\mathbb{O}}}^{G}(\mathcal{U}) \rightarrow \mathrm{HC}_{\text {fin }}^{Q}(\mathcal{W})$ has a right adjoint $\bullet \bullet^{\dagger}: \mathrm{HC}_{\text {fin }}^{Q}(\mathcal{W})$ $\rightarrow \mathrm{HC}_{\overline{\mathbb{O}}}(\mathcal{U})$. We will need the construction of the functor $\bullet{ }^{\dagger}$ below so let us recall it.

Pick $\underline{\mathcal{B}} \in \mathrm{HC}_{\text {fin }}^{Q}(\mathcal{W})$ and equip it with a $Q$-stable bimodule filtration (we can just take the trivial one). Then form the Rees bimodule $\underline{\mathcal{B}}_{\hbar}$ and the $Q$-equivariant $\mathcal{U}_{\hbar}^{\wedge \chi}$-bimodule $\mathcal{B}_{\hbar}^{\prime}=\mathbb{A}_{\hbar}^{\wedge_{0}} \widehat{\otimes}_{\mathbb{C}[\hbar]]} \underline{\mathcal{B}}_{\hbar}^{\wedge \chi}$. Now set

$$
\mathcal{F}_{G}\left(\mathcal{B}_{\hbar}^{\prime}\right):=\bigoplus_{V} V \otimes \operatorname{Hom}_{\mathfrak{g}}\left(V, \mathcal{B}_{\hbar}^{\prime}\right)
$$

where we view $\mathcal{B}_{\hbar}^{\prime}$ as a $\mathfrak{g}$-module with respect to the adjoint action, $\xi . b:=$ $\frac{1}{\hbar^{2}}[\xi, b]$, and the sum is taken over all $G$-modules $V$. In other words, $\mathcal{F}_{G}\left(\mathcal{B}_{\hbar}^{\prime}\right)$ is the maximal subspace in $\mathcal{B}_{\hbar}^{\prime}$, where the adjoint action of $\mathfrak{g}$ is locally finite and integrates to an action of $G$. The space $\mathcal{F}_{G}\left(\mathcal{B}_{\hbar}^{\prime}\right)$ is a $G$-equivariant bimodule over the algebra $\mathcal{U}_{\hbar}^{\diamond}$ that is the $\mathfrak{g}$-finite part of $\mathcal{U}_{\hbar}^{\wedge \hbar}$. Note that the subspace $\mathcal{F}_{G}\left(\mathcal{B}_{\hbar}^{\prime}\right)$ is $Q \times \mathbb{C}^{\times}$-stable in $\mathcal{B}_{\hbar}^{\prime}$. 
Lemma 3.2. $\mathcal{F}_{G}\left(\mathcal{B}_{\hbar}^{\prime}\right)$ is finitely generated as a left $\mathcal{U}_{\hbar}^{\diamond}$-bimodule.

Proof. We have $\mathcal{F}_{G}\left(\mathcal{B}_{\hbar}^{\prime}\right) / \hbar \mathcal{F}_{G}\left(\mathcal{B}_{\hbar}^{\prime}\right) \hookrightarrow \mathcal{F}_{G}\left(\mathcal{B}_{\hbar}^{\prime} / \hbar \mathcal{B}_{\hbar}^{\prime}\right)$. The latter is a finitely generated $\mathbb{C}\left[\mathfrak{g}^{*}\right]=\mathcal{U}_{\hbar}^{\diamond} /(\hbar)$-module by results of [17, Section 3.3]. So $\mathcal{F}_{G}\left(\mathcal{B}_{\hbar}^{\prime}\right) /$ $\hbar \mathcal{F}_{G}\left(\mathcal{B}_{\hbar}^{\prime}\right)$ is a finitely generated $\mathbb{C}\left[\mathfrak{g}^{*}\right]$-module as well. Pick $b$ lying in the $V$-isotypic component of $\mathcal{B}_{\hbar}^{\prime}$. The module $\mathcal{B}_{\hbar}^{\prime} / \hbar \mathcal{B}_{\hbar}^{\prime}$ is generated by finitely many elements $\underline{b}_{1}, \ldots, \underline{b}_{k}$ lying, say, in the components $V_{1}, \ldots, V_{k}$. Then we can argue by induction on $k$ to show that, modulo $\hbar^{k}$, the element $b$ is represented as $\sum_{i=1}^{k} a_{i}^{k} b_{i}$, where $b_{i}$ is a lift of $\underline{b}_{i}$ to the $V_{i}$-isotypic component of $\mathcal{B}_{\hbar}^{\prime}$, and $a_{i}^{k}$ lies in the sum of the isotypic components of $\mathcal{U}_{\hbar}^{\diamond}$ corresponding to summands of $V \otimes V_{i}^{*}$. Furthermore, we can achieve that the sequence of $a_{i}^{k}$ has limit for $k \rightarrow \infty$. This proves that the elements $b_{1}, \ldots, b_{k}$ generate $\mathcal{F}_{G}\left(\mathcal{B}_{\hbar}^{\prime}\right)$.

On $\mathcal{F}_{G}\left(\mathcal{B}_{\hbar}^{\prime}\right)$ we also have another action of $Q$ - one restricted from $G$. The two actions coincide on $Q^{\circ}$ and their difference is an action of $A:=Q / Q^{\circ}$ commuting with the $G$-action, see [17, Section 3.3]. Let $\mathcal{B}_{\hbar}$ denote the $\mathbb{C}^{\times}$finite part in $\mathcal{F}_{G}\left(\mathcal{B}_{\hbar}^{\prime}\right)^{A}$. Then we set $\underline{\mathcal{B}}^{\dagger}:=\mathcal{B}_{\hbar} /(\hbar-1)$. Again, $\underline{\mathcal{B}} \mapsto \underline{\mathcal{B}}^{\dagger}$ is a functor and one can show that it is right adjoint to $\bullet$. Moreover, the composition of $\bullet^{\dagger}$ with the quotient functor $\mathrm{HC}_{\overline{\mathbb{O}}}^{G}(\mathcal{U}) \rightarrow \mathrm{HC}_{\mathbb{O}}^{G}(\mathcal{U})$ is the left inverse of $\bullet_{\dagger}: \mathrm{HC}_{\mathbb{O}}(\mathcal{U}) \rightarrow \mathrm{HC}_{\text {fin }}^{Q}(\mathcal{W})$.

\subsection{Categories $\mathcal{O}$}

One can define the categories $\mathcal{O}$ for $\mathcal{W}$, see [5]. Namely, we have the quantum comoment map $\mathfrak{q} \rightarrow \mathcal{W}$, a Lie algebra homomorphism that can easily be shown to be an embedding, see [23, Section 2.1]. Pick a maximal torus $T \subset Q$ and a regular integral element $\theta \in \mathfrak{t}$. We define the category $\mathcal{O}_{\mathcal{W}}^{\theta}$ to be the full subcategory in the category of the finitely generated $\mathcal{W}$ modules consisting of all modules $M$ such that the real parts of eigenvalues are bounded from above and all generalized eigenspaces of $\theta$ are finite dimensional. This definition is easily seen to be equivalent to that in [5, Section 4.4].

In $\mathcal{O}_{\mathcal{W}}^{\theta}$ we have analogs of Verma modules. To define those we need some notation. The element $\theta$ defines a grading on $\mathcal{W}$, we have $\mathcal{W}=\bigoplus_{i} \mathcal{W}_{i}$, where $\mathcal{W}_{i}$ is the eigenspace of $[\theta, \cdot]$ with eigenvalue $i$. We set $\mathcal{W}_{\geqslant 0}:=\bigoplus_{i \geqslant 0} \mathcal{W}_{i}$ and $\mathcal{W}_{>0}:=\bigoplus_{i>0} \mathcal{W}_{i}$. Note that $\mathcal{W}_{>0}$ acts locally nilpotently on a module 
from $\mathcal{O}_{\mathcal{W}}^{\theta}$. The algebra $\mathcal{W}^{0}:=\mathcal{W}_{\geqslant 0} /\left(\mathcal{W}_{\geqslant 0} \cap \mathcal{W} \mathcal{W}_{>0}\right)$ acts on the annihilator $M^{\mathcal{W}>0}$. One can show that $M^{\mathcal{W}>0}$ is a finite dimensional $\mathcal{W}^{0}$-module, this is an easy consequence of [5, Corollary 4.12]. We have the Verma module functor $\Delta^{\theta}: \mathcal{W}^{0}-\bmod _{f g} \rightarrow \mathcal{O}_{\mathcal{W}}^{\theta}$ given by $\Delta^{\theta}(N):=\mathcal{W} / \mathcal{W} \mathcal{W}_{>0} \otimes_{\mathcal{W}^{0}} N$, it is left adjoint to $M \mapsto M^{\mathcal{W}>0}$.

There is a bijection between the irreducible $\mathcal{W}^{0}$-modules and the irreducible objects in $\mathcal{O}_{\mathcal{W}}^{\theta}$ : we send an irreducible $\mathcal{W}^{0}$-module $N$ to the maximal proper quotient $L^{\theta}(N)$ of $\Delta^{\theta}(N)$.

One can describe the algebra $\mathcal{W}^{0}$. Namely, consider the W-algebra $\underline{\mathcal{W}}$ for the pair $\left(\mathfrak{g}_{0}, e\right)$, where $\mathfrak{g}_{0}$ is the centralizer of $\theta$ in $\mathfrak{g}$. Then there is a $T$-equivariant filtered algebra isomorphism $\iota: \mathcal{W}^{0} \stackrel{\sim}{\rightarrow} \underline{\mathcal{W}}$, see [므, Theorem 4.3]. An important feature of this isomorphism is its behavior on $\mathfrak{t}$. Namely, we have $\iota(\xi)=\xi+\langle\delta, \xi\rangle$, where $\delta$ is half the character of the action of $\mathfrak{t}$ on $\Lambda^{t o p} V_{>0}$, the sum of the $\theta$-eigenspaces in $V$ with positive eigenvalues.

Below we will only need to know $\delta$ for birationally rigid orbits up to adding a character of a maximal torus $T \subset Q$.

Lemma 3.3. Assume that $G$ is simply connected and $(\mathbb{O}$ is birationally rigid. Suppose that $\mathfrak{g}_{0}$ is a standard Levi subalgebra and that the Lie algebra $\mathfrak{t}$ of $T$ is the center of $\mathfrak{g}_{0}$. Then up to adding a character of $T, \delta$ coincides with half the sum of the positive roots $\alpha$ satisfying $\langle\alpha, h\rangle=1$.

Proof. We may assume that $\theta$ is dominant. Note that $V_{>0} \oplus \mathfrak{z}_{\mathfrak{g}}(e)_{>0}=$ $\mathfrak{g}_{>0}$. Since $G$ is simply connected, an integral element in $\mathfrak{t}$ is the same as a restriction of an element in the weight lattice to $t$. Hence the character of the $T$-action on $\Lambda^{\text {top }} \mathfrak{g}_{>0}$ is divisible by 2 . Note further that $\mathfrak{z}_{\mathfrak{g}}(e) \cong \mathfrak{g}(0) \oplus \mathfrak{g}(1)$ as a $T$-module, where we write $\mathfrak{g}(i)$ for the eigenspace for $[h, \cdot]$ with eigenvalue $i$. So, to establish the claim of the lemma it is enough to check that the character of $T$ on $\Lambda^{\text {top }}\left(\mathfrak{g}(0) \cap \mathfrak{g}_{>0}\right)$ is divisible by 2 . For this, we observe that $(G(0), G(0))$ is simply connected because $G$ is simply connected. Also as we have seen in the proof of Lemma 2.9], the group $Q$ has no invariants in $\mathfrak{q}^{*}$ or, equivalently, in $\mathfrak{q}$. Since $Q \subset G(0)$, we conclude that $Q^{\circ} \subset(G(0), G(0))$. Since $(G(0), G(0))$ is simply connected, the character of a maximal torus of $(G(0), G(0))$ on $\Lambda^{\text {top }}\left(\mathfrak{g}(0) \cap \mathfrak{g}_{>0}\right)$ is indeed divisible by 2 .

We will need to compute $\delta$ (up to adding an integral weight) in two cases. 
Example 3.4. Consider the orbit $A_{2}+A_{1}$ in $E_{7}$. We use the notation for (simple) roots in $E_{7}$ from the table section of [32]. There the dual Cartan space $\mathfrak{h}^{*}$ is the quotient of $\mathbb{C}^{8}$ (with basis $\epsilon_{1}, \ldots, \epsilon_{8}$ ) by $\sum_{i=1}^{8} \epsilon_{i}$. The invariant bilinear form on $\mathfrak{h}^{*}$ is given $\left(\epsilon_{i}, \epsilon_{j}\right)=-\frac{1}{8}$ for $i \neq j$ and $\left(\epsilon_{i}, \epsilon_{i}\right)=\frac{7}{8}$. The basis of simple roots is given by $\alpha_{i}=\epsilon_{i}-\epsilon_{i+1},(i<7), \alpha_{7}=\epsilon_{5}+\epsilon_{6}+\epsilon_{7}+\epsilon_{8}$.

For a minimal Levi containing $e$ we take the standard Levi with simple roots $\alpha_{1}=\epsilon_{1}-\epsilon_{2}, \alpha_{2}=\epsilon_{2}-\epsilon_{3}, \alpha_{6}=\epsilon_{6}-\epsilon_{7}$. So we can take $h=2 \epsilon_{1}-2 \epsilon_{3}+$ $\epsilon_{6}-\epsilon_{7}$. The positive roots of $E_{7}$ are of the form $\epsilon_{i}-\epsilon_{j}$, where $i<j<8$ or $i=8$ and $\epsilon_{i}+\epsilon_{j}+\epsilon_{k}+\epsilon_{8}$, where $i, j, k$ are pairwise different numbers less than 8 . There are twelve positive roots whose pairing with $h$ equals 1 :

$\epsilon_{1}-\epsilon_{6}, \epsilon_{8}-\epsilon_{7}, \epsilon_{i}-\epsilon_{7}, \epsilon_{1}+\epsilon_{i}+\epsilon_{7}+\epsilon_{8}$, where $i=2,4,5, \epsilon_{1}+\epsilon_{3}+\epsilon_{6}+$ $\epsilon_{8}, \epsilon_{1}+\epsilon_{3}+\epsilon_{6}+\epsilon_{8}, \epsilon_{i}+\epsilon_{j}+\epsilon_{6}+\epsilon_{8}$, where $i, j \in\{2,4,5\}$.

The sum of these roots equals $\kappa:=5 \epsilon_{1}+4 \epsilon_{2}+\epsilon_{3}+4 \epsilon_{4}+4 \epsilon_{5}+3 \epsilon_{6}-\epsilon_{7}+8 \epsilon_{8}$.

The intersection of $\mathfrak{t}$ with the coroot lattice has basis $\epsilon_{1}+\epsilon_{2}+\epsilon_{3}-$ $3 \epsilon_{8}, 3 \epsilon_{8}-\epsilon_{5}-\epsilon_{6}-\epsilon_{7}, 2 \epsilon_{8}-\epsilon_{6}-\epsilon_{7}, 4 \epsilon_{8}$. The values of these elements on $\kappa$ are equal $-14,18,14,16$. We deduce that $\frac{1}{2} \kappa$ is integral on $\mathfrak{t}$ (where the lattice is the intersection of $\mathfrak{t}$ with the coroot lattice).

Example 3.5. Now consider the orbit $A_{4}+2 A_{1}$ in $E_{8}$. We again use the notation from [32]. There $\mathfrak{h}^{*}$ is the quotient of $\mathbb{C}^{9}$ (with basis $\epsilon_{1}, \ldots, \epsilon_{9}$ ) by $\sum_{i=1}^{9} \epsilon_{i}$. The invariant bilinear form on $\mathfrak{h}^{*}$ is given $\left(\epsilon_{i}, \epsilon_{j}\right)=-\frac{1}{9}$ for $i \neq j$ and $\left(\epsilon_{i}, \epsilon_{i}\right)=\frac{8}{9}$. The basis of simple roots is given by $\alpha_{i}=\epsilon_{i}-\epsilon_{i+1},(i<$ 8), $\alpha_{8}=\epsilon_{6}+\epsilon_{7}+\epsilon_{8}$.

For a minimal Levi containing $e$, we take the standard Levi subalgebra with simple roots $\alpha_{1}=\epsilon_{1}-\epsilon_{2}, \alpha_{2}=\epsilon_{2}-\epsilon_{3}, \alpha_{3}=\epsilon_{3}-\epsilon_{4}, \alpha_{4}=\epsilon_{4}-\alpha_{5}, \alpha_{7}=$ $\epsilon_{7}-\epsilon_{8}, \alpha_{8}=\epsilon_{6}+\epsilon_{7}+\epsilon_{8}$. So we can take $h=4 \epsilon_{1}+2 \epsilon_{2}-2 \epsilon_{4}-4 \epsilon_{5}+\epsilon_{6}+2 \epsilon_{7}$. The positive roots for $E_{8}$ are of the form $\epsilon_{i}-\epsilon_{j}, 1 \leqslant i<j \leqslant 9, \epsilon_{i}+\epsilon_{j}+\epsilon_{k}$, where $i, j, k \in\{1,2, \ldots, 8\}$ are pairwise distinct, $-\epsilon_{i}-\epsilon_{j}-\epsilon_{9}$, where $1 \leqslant i<j<9$. There are 14 positive roots that pair by 1 with $h$ :

$$
\begin{array}{r}
\epsilon_{6}-\epsilon_{8}, \epsilon_{6}-\epsilon_{9}, \epsilon_{2}-\epsilon_{6}, \epsilon_{1}+\epsilon_{2}+\epsilon_{5}, \epsilon_{1}+\epsilon_{5}+\epsilon_{7}, \epsilon_{1}+\epsilon_{3}+\epsilon_{4}, \epsilon_{1}+\epsilon_{4}+\epsilon_{8}, \epsilon_{2}+\epsilon_{3}+ \\
\epsilon_{8}, \epsilon_{2}+\epsilon_{4}+\epsilon_{7}, \epsilon_{3}+\epsilon_{7}+\epsilon_{8},-\epsilon_{3}-\epsilon_{8}-\epsilon_{9},-\epsilon_{1}-\epsilon_{5}-\epsilon_{9},-\epsilon_{2}-\epsilon_{4}-\epsilon_{9},-\epsilon_{4}-\epsilon_{7}-\epsilon_{9} .
\end{array}
$$

The sum of these roots equals $\kappa:=2 \epsilon_{1}+2 \epsilon_{2}+\epsilon_{3}+\epsilon_{7}-6 \epsilon_{9}$. A basis in the intersection of $\mathfrak{t}$ with the coroot lattice is given by the fundamental weights $\pi_{5}:=\sum_{i=1}^{5} \epsilon_{i}-5 \epsilon_{9}, \pi_{6}:=\sum_{i=1}^{6} \epsilon_{i}-3 \epsilon_{9}$. Their pairings with $\kappa$ are 35,23 . So we see that $\delta$ is not integral. 


\subsection{Classification of finite dimensional irreducible representations}

Here we will review results from [25] that concern the classification of finite dimensional irreducible $W$-algebra modules with integral central character.

Namely, recall that a primitive ideal $\mathcal{J}$ with associated variety $\overline{\mathbb{O}}$ exists if and only if $\mathbb{O}$ is special. Recall that the set of special nilpotent orbits is in one-to-one correspondence with the two-sided cells in $W$. To a two-sided cell c Lusztig assigned the quotient $\bar{A}$ of the component group $A=Q / Q^{\circ}$, see [26, p. 343]. One description of $\bar{A}$ is as follows. Consider the cell $W$ module $[\mathbf{c}]$ and the Springer $W \times A$-module $\operatorname{Spr}_{\mathbb{O}}$. Then $\bar{A}$ coincides with the quotient of $A$ by the kernel of the $A$-action on $\operatorname{Hom}_{W}\left([\mathbf{c}], \operatorname{Spr}_{\mathbb{O}}\right)$.

Now consider the primitive ideals with regular integral central character and associated variety $\mathbb{O}$. This set is in bijection with the set of left cells in c. When the character is not regular, the set of primitive ideals embeds into the set of left cells. To a left cell $\sigma \subset \mathbf{c}$ one can assign a subgroup $H_{\sigma} \subset \bar{A}$ that was first introduced in [27]. It can be defined as follows, see [25, Section 6]. Consider the $A$-module $\operatorname{Hom}_{W}\left([\sigma], \operatorname{Spr}_{\mathbb{O}}\right)$, where $[\sigma]$ denotes the cell module. Then $H_{\sigma}$ is a unique (up to conjugacy) subgroup in $\bar{A}$ such that the $\bar{A}$-module $\operatorname{Hom}_{W}\left([\sigma], \operatorname{Spr}_{\mathbb{O}}\right)$ is induced from the trivial $H_{\sigma}$-module.

Now take a primitive ideal $\mathcal{J}$ with associated variety $\overline{\mathbb{O}}$. The image $\mathcal{J}_{\dagger}$ is a maximal $Q$-stable ideal of finite codimension in $\mathcal{W}$. The simple finite dimensional modules annihilated by $\mathcal{J}_{\dagger}$ therefore form an $A$-orbit. It was checked in [16, Theorem 1.2.2] that every finite dimensional irreducible $\mathcal{W}$ module $N$ is annihilated by $\mathcal{J}_{\dagger}$ for some $\mathcal{J}$ as above that is forced to have the same central character as $N$. The main result of [25, Theorem 1.1] is that the $A$-orbit over a primitive ideal $\mathcal{J}$ with integral central character coincides with $\bar{A} / H_{\sigma}$, where $\sigma$ is the left cell corresponding to $\mathcal{J}$.

This implies the following corollary, see [25, Sections 6.5-6.8].

Corollary 3.6. Let $\mathbb{O}$ be a special orbit. Then the following is equivalent.

(a) $\mathcal{W}$ has no A-stable finite dimensional irreducible module with integral central character.

(b) $(1)$ is one of the orbits in (e2) of Section 1.2. 


\subsection{Quantizations of symplectic varieties}

Here $X$ is a smooth algebraic symplectic variety with form $\omega$. We assume that $X$ comes equipped with a $\mathbb{C}^{\times}$-action such that $t . \omega=t \omega$. We are going to recall the notion of a filtered quantization of $X$. By the conical topology on $X$, we mean a topology, where "open" means Zariski open and $\mathbb{C}^{\times}$-stable. In particular, the structure sheaf $\mathcal{O}_{X}$ can be viewed as a sheaf of graded algebras in the conical topology. By a filtered quantization $\mathcal{D}$ of $X$ we mean a sheaf of filtered algebras together with an isomorphism gr $\mathcal{D} \stackrel{\sim}{\rightarrow} \mathcal{O}_{X}$ of sheaves of graded Poisson algebras such that the filtration on $\mathcal{D}$ is complete and separated.

Now suppose that a reductive group $G$ acts on $X$ in a Hamiltonian way commuting with the $\mathbb{C}^{\times}$-action rescaling the form. Suppose that the $G$-action lifts to a filtration preserving action on $\mathcal{D}$. We say that the lifted action is Hamiltonian if there is a $G$-equivariant homomorphism $\Phi: \mathfrak{g} \rightarrow$ $\Gamma(\mathcal{D})$ such that $[\Phi(\xi), \cdot]$ coincides with the derivation induced by the $G$ action, for any $\xi \in \mathfrak{g}$. The map $\Phi$ automatically exists provided $H_{D R}^{1}(X)=$ 0 .

Consider the case when $X$ is a nilpotent orbit. Here $H_{D R}^{1}(X)=0$ by Lemma 2.9.

Lemma 3.7. Let $G$ be a semisimple group and $(\mathbb{O}$ its nilpotent orbit. There is a natural bijection between the following two sets.

(a) The set of quantizations of $\mathbb{O}$ with Hamiltonian G-action.

(b) The set of primitive ideals $\mathcal{J} \subset \mathcal{U}$ with associated variety $\overline{\mathbb{O}}$ and the multiplicity of $\mathcal{U} / \mathcal{J}$ on $\mathbb{O}$ is 1 .

A more general statement (that deals with coverings of nilpotent orbits) can be found in [31, Theorem 15], 20] (note that the set in (b) is in one-toone correspondence with the set of 1 -dimensional $A$-stable $\mathcal{W}$-modules). We provide an independent proof in our case.

Proof. Let $\mathcal{D}$ be a quantization. The map $\Phi$ extends to an algebra homomorphism $\mathcal{U} \rightarrow \Gamma(\mathcal{D})$. We have $\operatorname{gr} \Gamma(\mathcal{D}) \hookrightarrow \mathbb{C}[\mathbb{O}]$. Moreover, the composition of $\operatorname{gr} \Phi: \mathfrak{g} \rightarrow \operatorname{gr} \Gamma(\mathcal{D})$ with this inclusion coincides with the comoment map $\mu^{*}: \mathfrak{g} \rightarrow \mathbb{C}[\mathbb{O}]$. So the composition of $\operatorname{gr} \Phi: S(\mathfrak{g}) \rightarrow \operatorname{gr} \Gamma(\mathcal{D})$ with the inclusion $\operatorname{gr} \Gamma(\mathcal{D}) \hookrightarrow \mathbb{C}[\mathbb{O}]$ coincides with the map $\mu^{*}: S(\mathfrak{g}) \rightarrow \mathbb{C}[\mathbb{O}]$. Set 
$\mathcal{J}_{\mathcal{D}}:=\operatorname{ker} \Phi$. We have gr $\mathcal{J}_{\mathcal{D}} \subset \operatorname{ker} \mu^{*}$, the latter coincides with the ideal of $\overline{\mathbb{O}}$. In particular, $\overline{\mathbb{O}}$ is contained in the associated variety of $\mathcal{J}_{\mathcal{D}}$. On the other hand, we see that

$$
\operatorname{im} \mu^{*} \subset \operatorname{gr}\left(\mathcal{U} / \mathcal{J}_{D}\right) \subset \operatorname{gr} \Gamma(\mathcal{D}) \subset \mathbb{C}[\mathbb{O}]
$$

where the filtration on $\mathcal{U} / \mathcal{J}_{D}$ is induced from $\Gamma(\mathcal{D})$. The algebra $\mathbb{C}[\mathbb{O}]$ is finite over $\operatorname{im} \mu^{*}=\mathbb{C}[\overline{\mathbb{O}}]$. It follows that $\operatorname{gr} \Gamma(\mathcal{D})$ is finitely generated and the GK-dimension equals $\operatorname{dim} \mathbb{O}$. So $\Gamma(\mathcal{D})$ is a $\mathrm{HC}$ bimodule. From the inclusion $\mathcal{U} / \mathcal{J}_{\mathcal{D}} \hookrightarrow \Gamma(\mathcal{D})$ we deduce that the associated variety of $\mathcal{J}_{\mathcal{D}}$ is exactly $\overline{\mathbb{O}}$. Also from here we see that the multiplicity is 1 . Further, the algebra $\Gamma(\mathcal{D})$ has no zero divisors. It follows that $\mathcal{J}_{D}$ is a completely prime ideal. So it is primitive.

Given $\mathcal{J}$ as in (b), we can get a quantization $\mathcal{D}$ as in (a) by microlocalizing $\mathcal{U} / \mathcal{J}$ to the open subvariety $\mathbb{O} \subset \operatorname{Spec}(\operatorname{gr}(\mathcal{U} / \mathcal{J}))$. Let us denote the resulting quantization by $\mathcal{D}_{\mathcal{J}}$.

Now let us check that the maps $\mathcal{J} \mapsto \mathcal{D}_{\mathcal{J}}$ and $\mathcal{D} \rightarrow \mathcal{J}_{\mathcal{D}}$ are dual to one another. First of all, $\Phi$ factors through $\mathcal{U} / \mathcal{J}_{\mathcal{D}} \rightarrow \Gamma(\mathcal{D})$. This gives rise to a homomorphism $\mathcal{D}_{\mathcal{J}_{\mathcal{D}}} \rightarrow \mathcal{D}$ of filtered sheaves of algebras. From (3.2), we see that on the level of associated graded sheaves, this homomorphism is the identity. So $\mathcal{D}_{\mathcal{J}_{\mathcal{D}}} \stackrel{\sim}{\rightarrow} \mathcal{D}$.

On the other hand, we see that $\mathcal{J} \subset \mathcal{J}_{\mathcal{D}_{\mathcal{J}}}$. Since both ideals are prime and have the same associated variety, the equality $\mathcal{J}=\mathcal{J}_{\mathcal{D}_{\mathcal{J}}}$ follows now from [3, Corollary 3.6].

Now let us assume that $H^{1}\left(X, \mathcal{O}_{X}\right)=H^{2}\left(X, \mathcal{O}_{X}\right)=0$. In this case there is classification of quantizations, see [2, 21]. Namely, there is a natural bijection between the set of isomorphism classes of filtered quantizations of $X$ and $H_{D R}^{2}(X)$, see [21, Section 2.3]. If a reductive group $G$ is connected, then the $G$-action lifts to any filtered quantization, see the proof of [2, Proposition $6.2]$. 


\section{Proofs of the Main Results}

\section{1. $Q$-equivariance}

Here we will prove two results that give sufficient conditions for a $\mathfrak{q}$ action on a finite dimensional $\mathcal{W}$-module $V$ to integrate to an action of $Q^{\circ}$.

Lemma 4.1. Let $V$ be a 1 -dimensional $Q$-stable $\mathcal{W}$-module. Assume $\mathbb{O}$ is birationally rigid. Then the action of $\mathfrak{q}$ on $V$ is trivial.

So the action of $\mathfrak{q}$ on $V$ obviously integrates to an action of $Q^{\circ}$.

Proof. The proof of Lemma 2.9 shows that $\left(\mathfrak{q}^{*}\right)^{Q}=\{0\}$. Let $\chi$ be a character of the $\mathfrak{q}$-action on $V$. Since $V$ is $Q$-stable, we see that $\chi=q \cdot \chi$ for any $q \in Q$ so $\chi$ is $Q$-stable. It follows that $\chi$ is zero.

Proposition 4.2. Let $G$ be simply connected. Let $V$ be an irreducible $\mathcal{W}$ module with integral central character. Then the $\mathfrak{q}$-action on $V$ integrates to $a Q^{\circ}$-action if and only if the character $\delta$ recalled in Section 3.3 is integral.

Proof. Let us pick a maximal torus $T \subset Q^{\circ}$ and let $\mathfrak{t} \subset \mathfrak{q}$ be its Lie algebra. What we need to show is that $\mathfrak{t}$ acts diagonalizably and with characters lying in the character lattice of $T$. Pick a regular integral element $\theta \in \mathfrak{t}$. Let $\mathfrak{g}_{0}, \mathcal{W}^{0}$ be as in Section 3.3. Let us write $G_{0}$ for the connected subgroup of $G$ corresponding to $\mathfrak{g}_{0}$. Note that $T=Z\left(G_{0}\right)^{\circ}$. Since the group $G$ is simply connected, we see that the character group of $T$ is spanned by the fundamental weights that vanish on the roots for $\mathfrak{g}_{0}$.

We have $V=L_{\mathcal{W}}^{\theta}\left(V^{0}\right)$, where $V^{0}$ is an irreducible $\mathcal{W}^{0}$-module. Our claim reduces to checking that the character of the $\mathfrak{t}$-action on $V^{0}$ is integral.

Now recall the isomorphism $\mathcal{W}^{0} \stackrel{\sim}{\rightarrow} \underline{\mathcal{W}}$ that induces the shift by $\delta$ on t. Let $\underline{V}$ be the $\underline{\mathcal{W}}$-module corresponding to $V^{0}$. According to $[5$, Corollary 4.8], this module has integral central character. It follows that $\mathfrak{t}$ acts on $\underline{V}$ with integral character, i.e., it action integrates to $T$. So the action of $\mathfrak{t}$ on $V^{0}$ integrates to $T$ if and only if $\delta$ is integral.

Corollary 4.3. Let $\mathbb{O}$ be birationally rigid and special. Then the following are equivalent.

(1) $\mathcal{W}$ has an A-stable 1-dimensional module with integral central character. 
(2) $\delta$ is integral.

In particular, we see that $\delta$ is integral when $\mathfrak{g}$ is classical, [23, Section 5.4], or when $\mathbb{O}$ is, in addition, rigid, [38, Theorem B]. We remark that checking the integrality of $\delta$ involves only an elementary combinatorics and can be done in all these cases directly.

\subsection{Equivalence theorem}

Theorem 4.4. Suppose that $\operatorname{codim}_{\overline{\mathbb{O}}} \partial \mathbb{O} \geqslant 4$. Then the functor $\bullet_{\uparrow}: \mathrm{HC}_{\mathbb{O}}^{G}(\mathcal{U})$ $\rightarrow \mathrm{HC}_{\text {fin }}^{Q}(\mathcal{W})$ is a category equivalence.

We will need to introduce some auxiliary categories related to categories of $\mathrm{HC}$ bimodules. Recall that we write $\mathcal{U}_{\hbar}^{\diamond}$ for the $\mathfrak{g}$-finite part of $\mathcal{U}_{\hbar}^{\wedge}$. We write $\mathrm{HC}^{G}\left(\mathcal{U}_{\hbar}^{\diamond}\right)$ for the category of $G$-equivariant finitely generated $\mathcal{U}_{\hbar}^{\diamond}$ modules. Note that any such module $\mathcal{B}_{\hbar}$ becomes a $\mathcal{U}_{\hbar}$-bimodule by the formula $b \xi=\xi b-\hbar^{2} \xi . b$, where $b \in \mathcal{B}_{\hbar}, \xi \in \mathfrak{g}$ and $\xi . b$ is the image of $b$ under the derivation coming from the $G$-action. We write $\widetilde{\mathrm{HC}}^{G}\left(\mathcal{U}_{\hbar}^{\diamond}\right)$ for the ind completion of $\mathrm{HC}^{G}\left(\mathcal{U}_{\hbar}^{\diamond}\right)$, it consists of all $G$-equivariant $\mathcal{U}_{\hbar}^{\diamond}$-modules. Next, we consider the category $\operatorname{HC}\left(\mathcal{U}_{\hbar}^{\wedge} \chi\right)$ consisting of the $\mathfrak{g}$-equivariant finitely generated $\mathcal{U}_{\hbar}^{\wedge \chi}$-modules. We also consider the subcategories $\operatorname{HC}_{\overline{\mathbb{O}}}^{G}\left(\mathcal{U}_{\hbar}^{\diamond}\right) \subset$ $\mathrm{HC}^{G}\left(\mathcal{U}_{\hbar}^{\diamond}\right)$ of all modules supported on $\overline{\mathbb{O}}$ and $\mathrm{HC}_{\mathbb{O}^{\wedge} \chi}\left(\mathcal{U}_{\hbar}^{\wedge \chi}\right) \subset \mathrm{HC}\left(\mathcal{U}_{\hbar}^{\wedge \chi}\right)$ of all modules supported on $\mathbb{O}^{\wedge} \chi$.

Considering the functor $\mathcal{F}=\mathcal{F}_{G}: \mathrm{HC}_{\mathbb{O}^{\wedge} \chi}\left(\mathcal{U}_{\hbar}^{\wedge \chi}\right) \rightarrow \mathrm{HC}_{\overline{\mathbb{O}}}^{G}\left(\mathcal{U}_{\hbar}^{\diamond}\right)$ of taking $G$-finite sections. It extends a similar functor considered in Section 3.2 and it is given by

$$
\mathcal{B}_{\hbar}^{\prime} \mapsto \bigoplus_{V} V \otimes \operatorname{Hom}_{U(\mathfrak{g})}\left(V, \mathcal{B}_{\hbar}^{\prime}\right)
$$

where the sum is taken over all irreducible finite dimensional $G$-modules $V$. So the functor $\mathcal{F}$ admits derived functors

$$
R^{i} \mathcal{F}: \mathcal{B}_{\hbar}^{\prime} \mapsto \bigoplus_{V} V \otimes \operatorname{Ext}_{U(\mathfrak{g})}^{i}\left(V, \mathcal{B}_{\hbar}^{\prime}\right): \operatorname{HC}_{\mathbb{O}^{\wedge} \chi}\left(\mathcal{U}_{\hbar}^{\wedge} \chi\right) \rightarrow \widetilde{\mathrm{HC}}^{G}\left(\mathcal{U}_{\hbar}^{\diamond}\right)
$$

Here is the main technical result that is needed to establish Theorem 4.4. 
Lemma 4.5. Let $\mathcal{B}_{\hbar}^{\prime} \in \mathrm{HC}_{\mathbb{O}^{\wedge} \chi}\left(\mathcal{U}_{\hbar}^{\wedge \chi}\right)$ and $B:=\mathcal{B}_{\hbar}^{\prime} / \hbar \mathcal{B}_{\hbar}^{\prime}$. Then the following is true

(1) all $\mathfrak{g}$-isotypic components in $R^{1} \mathcal{F}\left(\mathcal{B}_{\hbar}^{\prime}\right)$ are finitely generated $\mathbb{C}[[\hbar]]$ modules.

(2) $R^{1} \mathcal{F}\left(\mathcal{B}_{\hbar}^{\prime}\right)$ is a finitely generated left $\mathcal{U}_{\hbar}^{\diamond}$-module and $R^{1} \mathcal{F}\left(\mathcal{B}_{\hbar}^{\prime}\right) / \hbar R^{1} \mathcal{F}\left(\mathcal{B}_{\hbar}^{\prime}\right)$ is supported on $\partial 0$.

(3) The cokernel of the natural homomorphism $\mathcal{F}\left(\mathcal{B}_{\hbar}^{\prime}\right) / \hbar \mathcal{F}\left(\mathcal{B}_{\hbar}^{\prime}\right) \hookrightarrow \mathcal{F}(B)$ is supported on $\partial \mathbb{O}$.

Proof. The proof is in several steps. Let us write $\tilde{G}$ for the simply connected cover of $G$.

Step 1 . We start by computing $R^{i} \mathcal{F}(M)$ for certain $M \in \mathrm{HC}_{\overline{\mathbb{O}}^{\wedge} \chi}\left(\mathcal{U}_{\hbar}^{\wedge \chi}\right)$. We will consider the objects annihilated by both $\hbar$ and the ideal of $\mathbb{O}$. The category of such objects was shown in [17, Section 3.2] to be equivalent to the category of $Z_{\tilde{G}}(e)^{\circ}$-modules: an object $N \in Z_{\tilde{G}}(e)^{\circ}$-mod gets sent to the sections $\mathcal{V}_{N}^{\wedge \chi}$ of the vector bundle $\mathcal{V}_{N}=\tilde{G} *_{Z_{\tilde{G}}}(e)^{\circ} N$ on the formal neighborhood of $Z_{\tilde{G}}(e)^{\circ}$ in $\tilde{G} / Z_{\tilde{G}}(e)^{\circ}$. Moreover, it was computed in [17, Section 3.2] that $\mathcal{F}\left(\mathcal{V}_{N}^{\wedge}\right)=\Gamma\left(\mathcal{V}_{N}\right)$. By the uniqueness of classical derived functors (where the source category is that of rational $Z_{\tilde{G}}(e)^{\circ}$-modules), we see that $R^{i} \mathcal{F}\left(\mathcal{V}_{N}^{\wedge \chi}\right)=H^{i}\left(\mathcal{V}_{N}\right)$ for all $i$ (all global sections and cohomology are taken on $\left.\tilde{\mathbb{O}}:=\tilde{G} / Z_{\tilde{G}}(e)^{\circ}\right)$.

Step 2. Let us prove that $H^{1}\left(\mathcal{V}_{N}\right)$ is a finitely generated $\mathbb{C}[\tilde{\mathbb{O}}]$-module supported on $\partial \tilde{\mathbb{O}}:=X \backslash \tilde{\mathbb{O}}$, where we write $X$ for $\operatorname{Spec}(\mathbb{C}[\tilde{\mathbb{O}}])$. Set $V_{N}:=\Gamma\left(\mathcal{V}_{N}\right)$, this is a coherent sheaf on $X$ whose restriction to $\tilde{\mathbb{O}}$ coincides with $\mathcal{V}_{N}$, see 17, Section 3.2]. Then $H^{i}\left(\mathcal{V}_{N}\right)=H_{\partial \tilde{\mathbb{O}}}^{i+1}\left(V_{N}\right)$. By [13, Expose VIII, Cor. 2.3], $H_{\partial \tilde{\mathbb{O}}}^{i+1}\left(V_{N}\right)$ is finitely generated (and obviously supported on $\partial \tilde{\mathbb{O}}$ ) provided $i+1<\operatorname{codim}_{X} \partial \tilde{\mathbb{O}}$. The right hand side in this inequality is at least $\operatorname{codim}_{\overline{\mathbb{O}}} \partial \mathbb{O}$ that is bigger than or equal to 4 by the assumptions of the proposition. The claim in the beginning of this step follows.

Step 3. Since any object $B$ in $\operatorname{HC}\left(\mathcal{U}_{\hbar}^{\wedge} \chi\right)$ annihilated by $\hbar$ admits a finite filtration by objects of the form $\mathcal{V}_{N}^{\wedge \chi}$, we deduce from Step 2 that for any such $B$, the $\mathbb{C}\left[\mathfrak{g}^{*}\right]$-module $R \mathcal{F}^{1}(B)$ is finitely generated and is supported on $\partial \mathbb{O}$. 
Step 4. In this step we prove (1). Set $B:=\mathcal{B}_{\hbar}^{\prime} / \hbar \mathcal{B}_{\hbar}^{\prime}$. Note that, for any finite dimensional $\mathfrak{g}$-module $V$, we have $\operatorname{dim}_{\operatorname{Ext}_{U(\mathfrak{g})}^{1}}^{1}(V, B)<\infty$. Indeed, the latter is the $V$-isotypic component in the finitely generated module $R^{1} \mathcal{F}(B)$ over $A:=\mathbb{C}\left[\mathfrak{g}^{*}\right] / \operatorname{Ann}(B)$. The reduced spectrum of $A$ is $\overline{\mathbb{O}}$, therefore $A^{G}$ is finite dimensional. The inequality $\operatorname{dim} \operatorname{Ext}_{U(\mathfrak{g})}^{1}(V, B)<\infty$ follows from the observation that any $\mathfrak{g}$-isotypic component in a finitely generated $A$-module is a finitely generated $A^{G}$-module.

Let us complete the proof of $(1)$. The space $\operatorname{Hom}_{\mathfrak{g}}\left(V, R^{1} \mathcal{F}\left(\mathcal{B}_{\hbar}^{\prime}\right)\right)$ coincides with $\operatorname{Ext}_{U(\mathfrak{g})}^{1}\left(V, \mathcal{B}_{\hbar}^{\prime}\right)$. The Ext groups $\operatorname{Ext}_{U(\mathfrak{g})}^{1}\left(V, \mathcal{B}_{\hbar}^{\prime}\right)$ are computed using the Chevalley-Eilenberg complex. In this complex, all cochains are complete and separated over $\mathbb{C}[[\hbar]]$. Arguing as in the end of the proof of $[12$, Lemma 5.6.3], we conclude that $\operatorname{Ext}_{U(\mathfrak{g})}^{1}\left(V, \mathcal{B}_{\hbar}^{\prime}\right)$ is finitely generated over $\mathbb{C}[[\hbar]]$.

Step 5. Let us prove (2). We have the following exact sequence

$$
0 \rightarrow \mathcal{F}\left(\mathcal{B}_{\hbar}^{\prime}\right) / \hbar \mathcal{F}\left(\mathcal{B}_{\hbar}^{\prime}\right) \rightarrow \mathcal{F}(B) \rightarrow R^{1} \mathcal{F}\left(\mathcal{B}_{\hbar}^{\prime}\right) \stackrel{\hbar}{\rightarrow} R^{1} \mathcal{F}\left(\mathcal{B}_{\hbar}^{\prime}\right) \rightarrow R^{1} \mathcal{F}(B) .
$$

It follows from Step 3 that $R^{1} \mathcal{F}(B)$ is a finitely generated $\mathbb{C}\left[\mathfrak{g}^{*}\right]$-module that is supported on $\partial \mathbb{O}$ By Step $4, R^{1} \mathcal{F}\left(\mathcal{B}_{\hbar}^{\prime}\right)$ is the direct sum of finitely generated $\mathbb{C}[[\hbar]]$-modules. The same argument as in the proof of Lemma 3.2 shows that $R^{1} \mathcal{F}\left(\mathcal{B}_{\hbar}^{\prime}\right)$ is a finitely generated $\mathcal{U}_{\hbar}^{\diamond}$-module. Since $R^{1} \mathcal{F}(B)$ is supported on $\partial \mathbb{O}$, then so is $R^{1} \mathcal{F}\left(\mathcal{B}_{\hbar}^{\prime}\right)$.

Step 6. Let us prove (3). The algebra $\mathcal{U}_{\hbar}^{\diamond}$ is Noetherian, this is established similarly to Lemma 3.2. It follows that the kernel of $\hbar$ in $R^{1} \mathcal{F}\left(\mathcal{B}_{\hbar}^{\prime}\right)$ admits a finite filtration whose quotients are subquotients in $R^{1} \mathcal{F}\left(\mathcal{B}_{\hbar}^{\prime}\right) / \hbar R^{1} \mathcal{F}\left(\mathcal{B}_{\hbar}^{\prime}\right)$. Therefore the kernel of $\hbar$ is finitely generated over $\mathbb{C}\left[\mathfrak{g}^{*}\right]$ and is supported on $\partial 0$.

Proof of Theorem 4.4. We can modify the categories involved in Lemma 4.5 considering weakly $\mathbb{C}^{\times}$-equivariant modules for $\mathcal{U}_{\hbar}^{\diamond}$ and $Q$-equivariant and Kazhdan weakly $\mathbb{C}^{\times}$-equivariant bimodules for $\mathcal{U}_{\hbar}^{\wedge}$. Then we have the functor $\mathcal{F}_{G}^{\prime}(\bullet)=\mathcal{F}(\bullet)_{\mathbb{C}^{\times} \text {-fin }}^{A}$ and (2) and (3) of that lemma still hold (with the same proof or as formal corollaries of those claims). Now pick $\mathcal{B} \in \mathrm{HC}_{\text {fin }}^{Q}(\mathcal{W})$ and apply these claims to $\mathcal{B}_{\hbar}^{\prime}:=\mathbb{A}_{\hbar}^{\wedge_{0}} \widehat{\otimes}_{\mathbb{C}[[\hbar]]} R_{\hbar}\left(\underline{\mathcal{B}}_{\hbar}\right)^{\wedge} \chi$. From the construction of $\bullet, \bullet^{\dagger}$ in Section 3.2, we see that (2) and (3) yield mult $\mathcal{B}^{\dagger}=\operatorname{dim} \mathcal{B}$. It follows that $\bullet^{\dagger}$ is exact and faithful when viewed as a functor $\mathrm{HC}_{\text {fin }}^{Q}(\mathcal{W}) \rightarrow$ 
$\mathrm{HC}_{\mathbb{O}}(\mathcal{U})$. Also it follows that $\operatorname{dim}\left(\mathcal{B}^{\dagger}\right)_{\dagger}=\operatorname{dim} \mathcal{B}$. Since $\left(\bullet_{\bullet}, \bullet^{\dagger}\right)$ is an adjoint pair, we see that $\bullet \dagger$ is a quasi-inverse of $\bullet$.

\subsection{Consequences of the equivalence theorem}

Proof of Theorem 1.1. Proof of (1). Let $X$ be a $\mathbb{Q}$-factorial terminalization of $\operatorname{Spec}(\mathbb{C}[\mathbb{O}])$. Let $\mathcal{D}$ be a quantization of $X^{r e g}$. The group $\pi_{1}\left(X^{r e g}\right)$ is finite because it coincides with $\pi_{1}(\mathbb{O})$. It follows that $H_{D R}^{1}\left(X^{r e g}\right)=0$ and so the $G$-action on $\mathcal{D}$ is Hamiltonian. From $H^{1}\left(X^{r e g}, \mathcal{O}_{X^{r e g}}\right)=0$ it follows that $\operatorname{gr} \Gamma(\mathcal{D})=\mathbb{C}\left[X^{r e g}\right]=\mathbb{C}[\mathbb{O}]$. Since the $G$-action on $\Gamma(\mathcal{D})$ is Hamiltonian, we see that $\Gamma(\mathcal{D})$ is a Dixmier algebra.

Proof of (2). Recall, Lemma 2.9, that since $\mathbb{O}$ is birationally rigid, we get $H_{D R}^{2}(\mathbb{O})=0$. Since $\operatorname{codim}_{X^{r e g}} X^{r e g} \backslash \mathbb{O} \geqslant 2$, we see that $H_{D R}^{2}\left(X^{r e g}\right)=0$. It follows that $X^{r e g}$ has a unique quantization. This finishes the proof.

Proof of (3). As we have seen in Example 3.4 and Section 4.1, in all cases except (e2) and (e3), $\delta$ is integral. Pick a unique $A$-stable 1-dimensional representation $V$ of $\mathcal{W}$ and a finite dimensional representation $U$ of $\mathcal{W}$ with integral central character. By Lemma $4.1 V$ is $Q^{\circ}$-equivariant. By Proposition 4.2, $U$ is $Q^{\circ}$-equivariant. So $\operatorname{Hom}_{\mathbb{C}}(U, V)$ is a $Q^{\circ}$-equivariant $\mathcal{W}$-bimodule. Let $\mathcal{B}$ be the set of $Q^{\circ}$-equivariant maps from $Q$ to $\operatorname{Hom}_{\mathbb{C}}(U, V)$ (with respect to the action of $Q^{\circ}$ on $Q$ ) from the right. It is a $Q$-equivariant $\mathcal{W}$-bimodule. Consider $\mathcal{B}^{\dagger}$. By Theorem 4.4, this is a nonzero HC $\mathcal{U}$-bimodule. The central character on the right is integral and therefore so is integral character on the left.

For the three orbits in (e2), there are no $A$-stable irreducible finite dimensional representations with integral central characters by Corollary 4.3 . For the orbit in (e3), we have checked in Example 3.5 that $\delta$ is non-integral.

Lemma 4.6. Let $\mathbb{O}$ be a birationally rigid orbit but not one of the orbits in (e1). Then there is a unique primitive ideal $\mathcal{J} \subset \mathcal{U}$ with associated variety $\overline{\mathbb{O}}$ and multiplicity of $\mathcal{U} / \mathcal{J}$ equal to 1 .

This lemma also follows from [39, Theorem 4]. Premet has checked in [38] that for the six orbits listed in (e1) there are exactly two ideals $\mathcal{J}$ with specified properties. 
Proof. By Corollary 2.8, $H^{i}\left(\mathbb{O}, \mathcal{O}_{\mathbb{O}}\right)=0$ for $i=1,2$. Besides, $H_{D R}^{1}(\mathbb{O})=0$ and $H_{D R}^{2}(\mathbb{O})=0$ by Lemma 2.9. So there is a unique Hamiltonian quantization of $\mathbb{O}$. The claim of this lemma now follows from Lemma 3.7.

There is a conjectural recipe of how to compute a highest weight for the primitive ideal $\mathcal{J}$ (at least, under the additional assumption that $\mathbb{O}$ is special). Namely, let $\mathbb{O}^{\vee}$ be the dual orbit of $\mathbb{O}$ and let $h^{\vee}$ be a dominant representative of the semisimple element for the $\mathfrak{s l}_{2}$-triple for $\mathbb{O}^{\vee}$. Following [1], set $\lambda_{\mathbb{O}}:=\frac{1}{2} h^{\vee}$. Then an expectation is that $\lambda_{\mathbb{O}}$ is a highest weight for $\mathcal{J}$. This is true for classical types and special birationally rigid orbits, 30 , Corollary 5.19], and for special rigid orbits in exceptional types, 38, Theorem $\mathrm{B}]$. Note that for the orbit $A_{2}+A_{1}$ in $E_{7}$, the dual orbit is $E_{6}\left(a_{1}\right)$, which is even. So $\lambda_{\mathbb{O}}$ in this case is integral. We also remark that [1, Theorem III] gives a character formula (i.e., computes the multiplicities of the irreducibles finite dimensional $\mathfrak{g}$-modules) for $\mathcal{U} / I\left(\lambda_{\mathbb{O}}\right)$, where $I\left(\lambda_{\mathbb{O}}\right)$ denotes the annihilator of the irreducible module with highest weight $\lambda_{\mathbb{O}}$, which holds even if $\lambda_{\mathbb{O}}$ is non-integral. The multiplicities for $\mathbb{C}[\mathbb{O}]$ are also known thanks to the work of McGovern, [29]. So one could try to compare the two sets of multiplicities to check if $\mathcal{U} / I\left(\lambda_{\mathbb{O}}\right)$ is multiplicity free. However, this is far from being straightforward as the formulas are different.

Proof of Corollary 1.2, If $\mathbb{O}$ is not one of the orbits in (e2),(e3), then there is a primitive ideal $\mathcal{J}$ with integral central character and associated variety $\overline{\mathbb{O}}$ such that the multiplicity of $\mathcal{U} / \mathcal{J}$ on $\mathbb{O}$ is 1 . It follows that $\mathcal{W}$ has an $A$-stable 1-dimensional module with integral central character. The claim of the present corollary follows from [23, Theorem 1.3].

Consider the three orbits in (e2). Let $\mathbb{O}$ be induced from an orbit $\mathbb{O}^{\prime}$ in a Levi and let $\mathcal{W}^{\prime}$ denote the $\mathrm{W}$-algebra corresponding to $\mathbb{O}^{\prime}$. Recall the exact dimension preserving functor $\rho: \mathcal{W}^{\prime}-\bmod _{f d} \rightarrow \mathcal{W}-\bmod$, see [18, Theorem 1.2.1]. It maps modules with integral central character to modules with integral central character by [18, Corollary 6.4.2]. The four orbits in (e2),(e3) are induced from special orbits, this can be shown using arguments in [10, Sections $3.3,3.4]$. So for all four orbits there are $(A$-unstable $) \mathcal{W}$ modules with integral central characters. For the orbits in (e2) we are now done by [23, Theorem 1.3].

So there is just one orbit, $A_{4}+2 A_{1}$, where a precise relationship between the dimensions of irreducible $\mathcal{W}$-orbits and the Goldie ranks of primitive 
ideals (with integral central character) is still unknown. Here $\bar{A}=\mathbb{Z} / 2 \mathbb{Z}$. If the left cell $\sigma$ corresponding to a primitive ideal $\mathcal{J}$ satisfies $H_{\sigma}=\{1\}$, then the dimension coincides with the Goldie rank, this follows from the proof of [23, Theorem 1.2]. If $H_{\sigma}=\bar{A}$, then the ratio of the dimension by the Goldie rank is either 1 or 2 .

Proposition 4.7. Let $(\mathbb{O}$ be a birationally rigid special orbit. Then $A \cong \bar{A}$.

This proposition is basically a special case of the description of $\bar{A}$ given in [26, p.343]. An advantage of our approach is that it is conceptual.

Proof. We use the notation from Section 3.4. Fix a left cell $\sigma$. The number of irreducible HC bimodules whose left and right annihilators are the primitive ideal (with central character $\rho$ ) corresponding to $\sigma$ is equal to $\sigma \cap \sigma^{-1}=|\operatorname{Irr}(\bar{A})|$. Applying $\bullet$ to these irreducible HC bimodules, we get sheaves on $\bar{A} / H_{\sigma}$ whose fibers at $H_{\sigma}$ are modules over $H_{\sigma}$. But any representation $V$ of $A$ defines an endo-functor $\mathrm{HC}_{\text {fin }}^{Q}(\mathcal{W}) \rightarrow \mathrm{HC}_{\text {fin }}^{Q}(\mathcal{W})$ given by $V \otimes \bullet$. If $A \rightarrow \bar{A}$ has a kernel, then the image of $\bullet \nmid$ is not stable under all functors $V \otimes \bullet$.

\section{Acknowledgments}

I would like to thank George Lusztig, Sasha Premet and David Vogan for stimulating discussions. This work was partially supported by the NSF under grants DMS-1161584, DMS-1501558.

\section{References}

1. D. Barbasch, D. Vogan. Unipotent representations, Ann. Math., 121(1985), 41-110.

2. R. Bezrukavnikov and D. Kaledin. Fedosov quantization in the algebraic context, Moscow Math. J., 4 (2004), 559-592.

3. W. Borho and H. Kraft. Über die Gelfand-Kirillov-Dimension, Math. Ann., 220(1976), $1-24$.

4. T. Braden, N. Proudfoot and B. Webster, Quantizations of conical symplectic resolutions I: local and global structure. arXiv:1208.3863.

5. J. Brundan, S. Goodwin and A. Kleshchev, Highest weight theory for finite W-algebras. Int. Math. Res. Not. IMRN 2008, no. 15, Art. ID rnn051.

6. R. Carter. Finite groups of Lie type. Conjugacy Classes and Complex Characters, 2nd ed., John Wiley and sons, 1993. 
7. D. Collingwood and W. McGovern. Nilpotent Orbits in Semisimple Lie Algebras. Chapman and Hall, London, 1993.

8. W. de Graaf, A. Elashvili. Induced nilpotent orbits of the simple Lie algebras of exceptional type. arXiv:0905.2743, Georgian Mathematical Journal, 16:257-278 (2009).

9. B. Fu, A survey on symplectic singularities and symplectic resolutions, Ann. Math. Blaise Pascal, 13 (2006), no.2, 209-236.

10. B. Fu, On $\mathbb{Q}$-factorial terminalizations of nilpotent orbits. J. Math. Pures Appl. (9), 93 (2010), no.6, 623-635.

11. B. Fu, D. Juteau, P. Levy and E. Sommers. Generic singularities of nilpotent orbit closures. arXiv:1502.05770.

12. I. Gordon and I. Losev, On category $\mathcal{O}$ for cyclotomic rational Cherednik algebras, $J$. Eur. Math. Soc., 16 (2014), 1017-1079.

13. A. Grothendieck, Cohomologie locale de faisceaux cohérent et théorèmes de Lefschetz locaux et globaux, North Holland, 1968.

14. H. Kraft and C. Procesi, Closures of conjugacy classes of matrices are normal. Invent. Math., 53 (1979), no.3, 227-247.

15. H. Kraft and C. Procesi, On the geometry of conjugacy classes in classical groups, Comment. Math. Helv., 57 (1982), no.4, 539-602.

16. I. Losev, Quantized symplectic actions and $W$-algebras, J. Amer. Math. Soc., 23 (2010), 35-59.

17. I. Losev, Finite dimensional representations of W-algebras, Duke Math. J., 159(2011), no.1, 99-143.

18. I. Losev, Parabolic induction and 1-dimensional representations for W-algebras, $A d v$. Math., 226(2011), no. 6, 4841-4883.

19. I. Losev, Finite W-algebras. Proceedings of the International Congress of Mathematicians Hyderabad, India, 2010, 1281-1307.

20. I. Losev, Quantizations of nilpotent orbits vs 1-dimensional representations of Walgebras, arXiv:1004.1669.

21. I. Losev, Isomorphisms of quantizations via quantization of resolutions, Adv. Math., 231 (2012), 1216-1270.

22. Primitive ideals in W-algebras of type A, J. Algebra, 359 (2012), 80-88.

23. I. Losev, Dimensions of irreducible modules over W-algebras and Goldie ranks, Invent. Math., 200 (2015), no.3, 849-923.

24. I. Losev, Deformations of symplectic singularities and Orbit method. In preparation.

25. I. Losev and V. Ostrik, Classification of finite dimensional irreducible modules over W-algebras, Compos. Math., 150(2014), no.6, 1024-1076.

26. G. Lusztig. Characters of reductive groups over a finite field, Ann. Math. Studies 107, Princeton University Press, 1984. 
27. G. Lusztig, Leading coefficients of character values of Hecke algebras, Proc. Symp. Pure Math., 47, Amer. Math. Soc., 1987, 235-262.

28. G. Lusztig and N. Spaltenstein, Induced unipotent classes, J. London Math. Soc. (2), 19 (1979), 41-52.

29. W. McGovern. Rings of regular functions on nilpotent orbits and their covers, Invent Math., 97 (1989), no.1, 209-217.

30. W. McGovern. Completely prime maximal ideals and quantization, Mem. Amer. Math. Soc., 519 (1994).

31. C. Moeglin. Modèles de Whittaker et idéaux primitifs complètement premiers dans les algèbres enveloppantes, Math. Scand., 63 (1988), 5-35.

32. A. Onishchik and E. Vinberg, Lie groups and algebraic groups. Translated from the Russian and with a preface by D. A. Leites. Springer Series in Soviet Mathematics. Springer-Verlag, Berlin, 1990.

33. V. Ostrik, Multi-fusion categories of Harish-Chandra bimodules, arXiv:1404.6575.

34. Y. Namikawa, Flops and Poisson deformations of symplectic varieties, Publ. Res. Inst. Math. Sci., 44 (2008), no. 2, 259-314.

35. Y. Namikawa. Induced nilpotent orbits and birational geometry, Adv. Math.,

222 (2009), no.2, 547-564.

36. A. Premet. Special transverse slices and their enveloping algebras, Adv. Math., 170(2002), 1-55.

37. A. Premet. Enveloping algebras of Slodowy slices and the Joseph ideal, J. Eur. Math. Soc., 9(2007), no.3, 487-543.

38. A. Premet. Multiplicity-free primitive ideals associated with rigid nilpotent orbits, Transform. Groups, 19 (2014), no.2, 569-641.

39. A. Premet and L. Topley, Derived subalgebras of centralizers and finite W-algebras, Compos. Math., 150 (2014), 1485-1548.

40. D. Vogan, Dixmier algebras, sheets, and representation theory, Operator algebras, unitary representations, enveloping algebras, and invariant theory (Paris, 1989), 333395, Progr. Math., 92, Birkhuser Boston, Boston, MA, 1990.

41. W. Wang, Nilpotent orbits and W-algebras, arXiv:0912.0689. Fields Institute Communications Series, 59 (2011), 71-105. 